\title{
Addendum to "Termination of 4-fold Canonical Flips"
}

By

\author{
Osamu Fujino*
}

\begin{abstract}
There are errors in the definition of the weighted version of 'difficulty' in "Termination of 4-fold canonical flips". In this paper, we describe these errors and correct them. After these corrections, Theorem 5.2 holds: every sequence of 4-fold canonical flips terminates.
\end{abstract}

\section{$\S 1$. Introduction}

Professor Alexeev pointed out that Lemma 2.1 in [F1], which is a copy of $\left[\mathrm{K}^{+},(4.12 .2 .1)\right]$, is wrong. Therefore, the weighted version of difficulty $d_{S, b}(X, B)$ in Definition 2.3 in [F1] is infinite if $b<\max _{j}\left\{b_{j}\right\}$. So, the proof in [F1] is nonsense. In this paper, we change the definition of $d_{S, b}(X, B)$ to make it finite when $(X, B)$ is canonical and $B$ has no reduced components, that is, the round down $\llcorner B\lrcorner=0$. Roughly speaking, in [F1, Definition 2.3] we exclude valuations obtained by one blow-up along generic points of codimension two subvarieties when we count valuations with small discrepancies. In this paper, we exclude valuations whose centers are codimension two subvarieties with good properties. By this change, the new version of $d_{S, b}(X, B)$ defined in Definition 4.4 becomes finite and the arguments in [F1] work without any changes. Proposition 3.1 is a key result in this paper. Note that the problems in [F1] are not in the arguments but in the definitions. As mentioned above,

Communicated by S. Mori. Received May 31, 2004.

2000 Mathematics Subject Classification(s): Primary 14E05; Secondary 14J35.

*Graduate School of Mathematics, Nagoya University, Chikusa-ku Nagoya 464-8602, Japan.

e-mail: fujino@math.nagoya-u.ac.jp

(C) 2005 Research Institute for Mathematical Sciences, Kyoto University. All rights reserved. 
we have to assume $\llcorner B\lrcorner=0$ to make $d_{S, b}(X, B)$ finite. Thus the main theorem: Theorem 1.1 in [F1] becomes slightly weaker. However, this assumption is harmless for applications if we use the special termination theorem (see [F3]). For the precise statements of the termination theorems, see Theorems 5.1 and 5.2 below. Anyway, any sequence of 4-fold canonical flips terminates.

We summarize the contents of this paper. In Section 2, we describe the errors in discrepancy lemmas in $\left[\mathrm{K}^{+}, 4.12\right]$. In Section 3, we formulate a new discrepancy lemma. Proposition 3.1 is the main result in this paper. In Section 4 , we explain how to modify the definition of the weighted version of difficulty. Section 5 is devoted to the statements of the termination of 4 -fold canonical flips. We will use the same notation as in [F1] throughout this paper.

\section{§2. Errors in Discrepancy Lemmas}

The following example contradicts [F1, Lemma 2.1], which is a copy of $\left[\mathrm{K}^{+},(4.12 .2 .1)\right]$.

Example 2.1. Let $X=\mathbb{P}^{2}, B=\frac{2}{3} L$, where $L$ is a line on $X$. Let $P$ be any point on $L$. First, blow up $X$ at $P$. Then we obtain an exceptional divisor $E_{P}$ such that $a\left(E_{P}, X, B\right)=\frac{1}{3}$. Let $L^{\prime}$ be the strict transform of $L$. Next, take a blow-up at $L^{\prime} \cap E_{P}$. Then we obtain an exceptional divisor $F_{P}$ whose discrepancy $a\left(F_{P}, X, B\right)=\frac{2}{3}$. Note that this $F_{P}$ is essential in the notation in $\left[\mathrm{F} 1\right.$, Definition 2.1]. On the other hand, it is easy to see that $\operatorname{discrep}(X, B)=\frac{1}{3}$. Thus, $\min \{1,1+\operatorname{discrep}(X, B)\}=1$.

The proof of $\left[\mathrm{K}^{+},(4.12 .2 .1)\right]$ depends on $\left[\mathrm{K}^{+},(4.12 .1 .3)\right]$, which is obviously wrong by Example 2.1 above. We need some extra assumption. It is not difficult to see that $\left[\mathrm{K}^{+},(4.12 .1 .3)\right]$ is true if we assume that $b_{j} \leq \frac{1}{2}$ for all $j$. We write the precise statement for the reader's convenience. This is essentially the same as [K, Corollary 3.2 (iii)] (see Remark 2.5 below).

Lemma 2.2. $\quad$ Let $Y$ be a smooth variety with a (not necessarily effective) $\mathbb{Q}$-divisor $B=\sum_{i} b_{i} B_{i}$ such that $\sum_{i} B_{i}$ has simple normal crossings, $B_{i}$ is a prime divisor for every $i, B_{k} \neq B_{l}$ for $k \neq l$, and that $b_{i} \leq \frac{1}{2}$ for all $i$. Assume that $b_{k}+b_{l} \leq 0$ whenever $B_{k}$ and $B_{l}$ intersects. If $\nu$ is an algebraic valuation with small center on $Y$ such that $a(\nu, Y, B)<1$ then $\nu$ is obtained by blowing up the generic point of a subvariety $W \subset Y$ such that $\operatorname{codim}_{Y} W=2$, only one of the $B_{k}\left(\right.$ say $\left.B_{k_{0}}\right)$ contains $W$ and $b_{k_{0}}>0$.

Remark 2.3. In Example 2.1, we put $D=d L$. Then $a\left(E_{P}, X, D\right)=1-d$. Thus, the coefficient of $E_{P}$ (resp. $D^{\prime}$, the strict transform of $\left.D\right)$ is $d-1$ (resp. $d$ ). 
Thus, $(d-1)+d \leq 0$ if and only if $d \leq \frac{1}{2}$. This computation shows that we have to assume $b_{j} \leq \frac{1}{2}$ for all $j$ in Lemma 2.2 .

Thus we obtain the following lemma, which is a correction of $\left[\mathrm{K}^{+}\right.$, (4.12.2.1)]. The proof is an exercise. Note that $\left[\mathrm{K}^{+},(4.12 .2 .2)\right]$ is contained in [KM, Proposition $2.36(2)]$. We do not need $d_{j} \leq \frac{1}{2}$ for $\left[\mathrm{K}^{+},(4.12 .2 .2)\right]$.

Lemma 2.4. Let $X$ be a normal variety and $D=\sum_{j} d_{j} D_{j}$ an effective $\mathbb{Q}$-divisor on $X$ such that $K_{X}+D$ is $\mathbb{Q}$-Cartier, where $D_{j}$ is a prime divisor for every $j$ and $D_{k} \neq D_{l}$ for $k \neq l$. Assume that $\operatorname{discrep}(X, D) \geq-\frac{1}{2}$ and $d_{j} \leq \frac{1}{2}$ for all $j$. Let $\nu$ be an algebraic valuation with small center on $X$. Then there is a finite set of valuations $\left\{\nu_{i}\right\}$ such that if

$$
a(\nu, X, D)<\min \{1,1+\operatorname{discrep}(X, D)\} \text { and } \nu \notin\left\{\nu_{i}\right\}
$$

then $\nu$ is obtained from blowing up the generic point of a subvariety $W \subset D \subset X$ such that $D$ and $X$ are generically smooth along $W$ (and thus only one of the $D_{j}$ contains $W$ ) and $\operatorname{dim} W=\operatorname{dim} X-2$.

Unfortunately, Lemmas 2.2 and 2.4 are useless for our purpose. The assumption that $b_{j} \leq \frac{1}{2}$ for all $j$ is too strong. Proposition 3.1 below seems to be a better formulation.

Remark 2.5. Note that there are no problems in [K, Corollary 3.2 (iii)] since Kollár assumed $c>-\frac{1}{2}$ (for the notation, see Corollary 3.2 in $[\mathrm{K}]$ ). The assumption $c>-\frac{1}{2}$ is in [K, Corollary 3.2 (ii)]. Lemma 2.2 in [M] is almost an exact copy of Corollary 3.2 in $[\mathrm{K}]$. Therefore, [M, Lemma 2.2] is also correct. Matsuki gave me a comment about the remark which he made in [M, Lemma 2.2 (ii)] and which is not in [K, Corollary 3.2] "(actually $>-1$ is enough for the conclusion)". This has to be understood that if we have the assumption $0 \geq c>-1$, then the conclusion for (ii) holds (for the proof, see [KM, Proposition $2.36(2)]$ ), and NOT that the conclusion of (iii) holds (as Example 2.1 above is an obvious counter-example then). Thus, with the understanding that the assumptions are accumulative and not independent, it seems that the statements of the Corollary 3.2 in $[\mathrm{K}]$ and Lemma 2.2 in $[\mathrm{M}]$ are correct and that the proof does not need any modifications. Therefore, the problems are not in $[\mathrm{K}]$ nor in $[\mathrm{M}]$, but in $\left[\mathrm{K}^{+},(4.12 .1 .3)\right]$. For the finiteness of $d_{N}(X, D)$ in $\left[\mathrm{K}^{+}, 4.12 .3\right.$ Definition], we do not need $\left[\mathrm{K}^{+},(4.12 .2 .1)\right]$. The statement $\left[\mathrm{K}^{+},(4.12 .2 .2)\right]$, which is true by [KM, Proposition $\left.2.36(2)\right]$, is sufficient. So, the error in $\left[\mathrm{K}^{+},(4.12 .1 .3)\right]$ causes no serious troubles in $\left[\mathrm{K}^{+}\right.$, Chapter 4]. 


\section{§3. New Discrepancy Lemma}

The following proposition is a key result in this paper. The proof is essentially the same as one of $\left[\mathrm{K}^{+},(4.12 .2 .1)\right]$. We give a proof for the reader's convenience.

Proposition 3.1. $\quad$ Let $X$ be a normal variety and $B=\sum_{i} b_{i} B_{i}$ a $\mathbb{Q}$ divisor on $X$ with $\llcorner B\lrcorner \leq 0$, where $B_{i}$ is a prime divisor for every $i$ and $B_{k} \neq B_{l}$ for $k \neq l$. Assume that $K_{X}+B$ is $\mathbb{Q}$-Cartier and $\operatorname{discrep}(X, B)>-1$. Note that $(X, B)$ is called a sub klt pair in some literatures. Let $\nu$ be an algebraic valuation with small center on $X$. Then there is a finite set of valuations $\left\{\nu_{i}\right\}$ such that if

$$
a(\nu, X, D)<\min \{1,1+\operatorname{discrep}(X, D)\} \text { and } \nu \notin\left\{\nu_{i}\right\}
$$

then $V:=$ Center $_{X} \nu \subset B \subset X, B$ and $X$ are generically smooth along $V$, $\operatorname{dim} V=\operatorname{dim} X-2$, and only one of the $B_{k}$ (say $\left.B_{k_{0}}\right)$ contains $V$ and $b_{k_{0}}>0$.

Proof. First, we take a log resolution $f: Y \longrightarrow X$ as in [KM, Proposition 2.36]. Thus, we have $f^{*}\left(K_{X}+B\right)=K_{Y}+A-C$, where $A$ and $C$ are both effective divisors with the following properties:

(i) $A=\sum_{a_{i}>0} a_{i} A_{i}$ and $C=\sum_{c_{j} \geq 0} c_{j} C_{j}$ have no common irreducible components,

(ii) $\operatorname{Exc}(f) \cup \operatorname{Supp} f_{*}^{-1} B=\sum_{i} A_{i} \cup \sum_{j} C_{j}$, and

(iii) $\sum_{i} A_{i} \cup \sum_{j} C_{j}$ is a simple normal crossing divisor and $\sum_{i} A_{i}$ is smooth.

Note that $c_{j}$ may be zero and that $A=f_{*}^{-1} B+D$, where $D$ is an effective $\mathbb{Q}$-divisor such that $\operatorname{Supp} D \cap \operatorname{Supp} f_{*}^{-1} B=\emptyset$. Next, if $E$ is an exceptional divisor over $Y$ such that $a(E, Y, A-C)<1$, then $V:=$ Center $_{Y} E \subset A \subset Y$ and $\operatorname{dim} V=\operatorname{dim} Y-2$ by the following lemma: Lemma 3.2. We note that in general $a\left(E, Y, F^{\prime}\right) \leq a(E, Y, F)$ if $F^{\prime} \geq F$ for any valuation $E$. If $V$ is contained in $D$, then $a(E, Y, A-C) \geq 1+\operatorname{discrep}(X, B)$. Finally, the number of the exceptional divisors over $Y$ whose centers are in $f_{*}^{-1} B \cap C$ with $a(\cdot, Y, A-C)<1$ is finite (see Lemma 3.2 below), and it is obvious that the number of $f$-exceptional divisors is finite. Thus, we obtain the required finite set of valuations $\left\{\nu_{i}\right\}$.

Lemma 3.2. Let $Y$ be a smooth variety and $H=d P$, where $P$ is a smooth prime divisor on $Y$ and $0<d<1$. Then $\operatorname{discrep}(Y, H)=1-d$. If $a(E, Y, H)<1$ for an exceptional divisor $E$ over $Y$, then $\operatorname{Center}_{Y} E$ is a codimension two subvariety of $Y$ such that $\operatorname{Center}_{Y} E \subset P \subset Y$. 
Let $W$ be a codimension two subvariety of $Y$ such that $W \subset P \subset Y$. Then there are only finitely many algebraic valuations $\nu$ 's with the following properties:

(1) $a(\nu, Y, H)<1$,

(2) $\operatorname{Center}_{Y} \nu=W$.

Furthermore, $\nu$ attains the minimum, that is, $a(\nu, Y, H)=1-d$, if and only if $\nu$ is obtained by blowing up $Y$ along $W$.

Proof. This follows from easy computations. See [KM, Lemmas 2.45 and 2.29].

\section{§4. How to Define a Weighted Difficulty}

We introduce the notion of significant divisors. Proposition 3.1 and Lemma 2.2 imply that the notion of significant divisors are much better than one of essential divisors in [F1, Definition 2.3] for our purpose.

Definition 4.1. $\quad$ Let $(X, B)$ be a canonical pair. We say that an exceptional divisor $E$ (over $X$ ) is significant unless $W=\operatorname{Center}_{X} E$ is a subvariety $W \subset B \subset X$ such that $B$ and $X$ are generically smooth along $W$ (and thus only one of the irreducible components of $\operatorname{Supp} B$ contains $W$ ) and $\operatorname{dim} W=\operatorname{dim} X-2$.

The following corollary is obvious by Proposition 3.1. We will use this to define a weighted version of difficulty.

Corollary 4.2. Let $(X, B)$ be a canonical pair with $\llcorner B\lrcorner=0$. Then we have

$$
\sharp\{E \mid E \text { is significant and } a(E, X, B)<1\}<\infty .
$$

Remark 4.3. Let $(X, B)$ be a canonical pair. Assume that $\llcorner B\lrcorner \neq 0$. Let $f: Y \longrightarrow X$ be a $\log$ resolution of $(X, B)$ with $f^{*}\left(K_{X}+B\right)=K_{Y}+\sum_{i} a_{i} E_{i}$ such that $\sum_{i} E_{i}=\operatorname{Exc}(f) \cup \operatorname{Supp} f_{*}^{-1} B$. We can assume that $a_{0}=1$. If $E_{0}$ intersects $E_{1}$ such that $0 \leq a\left(E_{1}, X, B\right)=-a_{1}<1$ and $\operatorname{codim}_{X} f\left(E_{0} \cap E_{1}\right) \geq 3$, then we have infinitely many significant divisors whose centers are $f\left(E_{0} \cap E_{1}\right)$ with $a(\cdot, X, B)=-a_{1}$ by suitable blowing-ups whose centers are over $E_{0} \cap E_{1}$.

We define a weighted version of difficulty. To define this, we have to assume that the boundary divisor has no reduced components. 
Definition 4.4 (A weighted version of difficulty). Let $(X, B)$ be a pair with only canonical singularities, where $B=\sum_{j=1}^{l} b_{j} B^{j}$ with $0<b_{1}<\cdots<$ $b_{l}<1$ and $B^{j}$ is a reduced divisor for every $j$. We note that $B^{j}$ is not necessarily irreducible and that we assume $b_{l}<1$. If $(X, B)$ has only terminal singularities, then $\llcorner B\lrcorner=0$. Thus the assumption $b_{l}<1$ always holds for terminal pairs. We put $b_{0}=0$, and $S:=\sum_{j \geq 0} b_{j} \mathbb{Z}_{\geq 0} \subset \mathbb{Q}$. Note that $S=0$ if $B=0$. We set

$$
d_{S, b}(X, B):=\sum_{\xi \in S, \xi \geq b} \sharp\{E \mid E \text { is significant and } a(E, X, B)<1-\xi\} .
$$

Then $d_{S, b_{j}}(X, B)$ is finite by Corollary 4.2 .

\section{$\S 5$. Statements of the Termination Theorems}

Now the proof in $[F 1, \S 3]$ works without any changes only if we replace the word "essential" with "significant". Thus we obtain the following theorem, which is slightly weaker than the original theorem: Theorem 1.1 in [F1].

Theorem 5.1. $\quad$ Let $X$ be a normal projective 4-fold and $B$ an effective $\mathbb{Q}$-divisor such that $(X, B)$ is canonical and $\llcorner B\lrcorner=0$. Consider a sequence of log flips starting from $(X, B)=\left(X_{0}, B_{0}\right)$ :

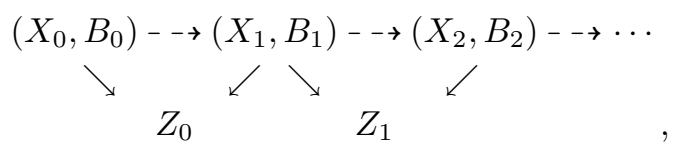

where $\phi_{i}: X_{i} \longrightarrow Z_{i}$ is a contraction and $\phi_{i}{ }^{+}: X_{i}^{+}=X_{i+1} \longrightarrow Z_{i}$ is the log flip. Then this sequence terminates after finitely many steps.

As we pointed out before, $\llcorner B\lrcorner=0$ if $(X, B)$ has only terminal singularities. Under the assumption that the varieties are $\mathbb{Q}$-factorial and all the flipping contractions have the relative Picard number one, we obtain the following theorem by using the special termination theorem. These assumptions are harmless for applications.

Theorem 5.2. $\quad$ Let $X$ be a normal projective 4 -fold and $B$ an effective $\mathbb{Q}$-divisor such that $(X, B)$ is canonical. Assume that $X$ is $\mathbb{Q}$-factorial. Consider a sequence of log flips starting from $(X, B)=\left(X_{0}, B_{0}\right)$ :

$$
\begin{aligned}
& \left(X_{0}, B_{0}\right) \rightarrow\left(X_{1}, B_{1}\right)-\rightarrow\left(X_{2}, B_{2}\right)-\rightarrow \cdots \\
& Z_{0} \quad Z_{1}
\end{aligned}
$$


where $\phi_{i}: X_{i} \longrightarrow Z_{i}$ is a contraction and $\phi_{i}{ }^{+}: X_{i}{ }^{+}=X_{i+1} \longrightarrow Z_{i}$ is the log flip. We further assume that the relative Picard number $\rho\left(X_{i} / Z_{i}\right)=1$ for every $i$. Then this sequence terminates after finitely many steps.

Proof. By applying the special termination theorem (see [F3]) and shifting the index, we can assume that the flipping and flipped loci are disjoint from $\left\llcorner B_{i}\right\lrcorner$ for every $i$. So, we can replace $B_{i}$ with its fractional part. Thus this sequence terminates by Theorem 5.1 .

Remark 5.3. The final remark in [F1] should be removed. In [F2], we only need the termination of 4 -fold semi-stable terminal flips. See Definition 2.3 in [F2]. Therefore, Theorems 5.1 and 5.2 are sufficient for [F2].

\section{Acknowledgements}

I would like to thank Professor Valery Alexeev, who pointed out an error in [F1], gave me comments, and obtained the same correction independently. This paper was written in the Institute for Advanced Study. I am grateful to it for its hospitality. I was partially supported by a grant from the National Science Foundation: DMS-0111298. After I wrote the preliminary version of this paper, I received many useful comments from Professor Kenji Matsuki. I would like to thank him.

\section{References}

[F1] Fujino, O., Termination of 4-fold canonical flips, Publ. RIMS, Kyoto Univ., 40 (2004), 231-237.

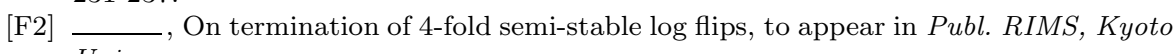
Univ.

[F3] Special termination and reduction theorem, to be contained in the book prepared by A. Corti et al.

[K] Kollár, J., Flops, Nagoya Math. J., 113 (1989), 15-36.

[KM] Kollár, J. and Mori, S. Birational geometry of algebraic varieties, Cambridge Tracts in Math., 134. Cambridge University Press, Cambridge, 1998.

$\left[\mathrm{K}^{+}\right]$Kollár, J., et al., Flips and Abundance for algebraic threefolds, Astérisque, 211 (1992).

[M] Matsuki, K., Termination of flops for 4-folds, Amer. J. Math., 113 (1991), 835-859. 